\title{
Numerical Treatment of the Rotation Number for the Forced Pendulum
}

\author{
Raffaella Pavani \\ Dipartimento di Matematica, Politecnico di Milano \\ 20133 Milano - ITALY \\ rafpav@mate.polimi.it
}

\begin{abstract}
The aim of this paper is to study (from a numerical point of view) the behavior of orbits of the forced pendulum. We resort mainly to the numerical method by the author, which allows to distinguish efficiently the numerical approximation of a rational rotation number from that one of an irrational rotation number, so we can characterize respectively the periodic and non-periodic behavior of the orbits. Moreover we study numerically the appearance of chaotic behavior depending on the forcing parameter and we show how the numerical results provide useful and reliable information about such an event.
\end{abstract}

\section{Introduction to the problem}

We consider the motion of a forced pendulum that moves on a vertical plane; in absence of friction, the displacement angle $\vartheta$ from the vertical rest position of the pendulum satisfies the following second order differential equation:

$$
\frac{d^{2} \vartheta}{d t^{2}}+\frac{g}{l} \alpha(t) \sin \vartheta=0
$$

where $g$ is the gravitational constant and $l$ is the length of the pendulum; without loss of generality now we assume $g=l$; moreover we assume $\alpha(t)=1+\varepsilon \cos t$, where $\varepsilon$ is the forcing parameter and is supposed to be very small (i.e. $\varepsilon<<1$ ). Introducing the variables

$$
x_{1}(t)=\vartheta(t), \quad x_{2}(t)=\frac{d \vartheta}{d t}
$$

equation (1) becomes

$$
\begin{aligned}
& \frac{d x_{1}}{d t}=x_{2} \\
& \frac{d x_{2}}{d t}=-(1+\varepsilon \cos t) \sin x_{1}
\end{aligned}
$$

At first we assume $\varepsilon=0$.

We are interested in the orbits of the pendulum away from the equilibrium point $(n \pi, 0)$, where $n$ is any integer point. As $x_{1}$ is $2 \pi$-periodic, we will consider 
the vertical strip of the plane with $0 \leq x_{1} \leq 2 / p i$. Moreover for the same reason, it suffices to take initial data on the $x_{2}$-axis, say $x_{2}^{0}$. Therefore we need to determine the shapes of the curves of the form

$$
\frac{1}{2}\left(x_{2}\right)^{2}+\left(1-\cos x_{1}\right)=\frac{1}{2}\left(x_{2}^{0}\right)^{2}
$$

where $x_{2}^{0}$ is the initial data on the $x_{2}$-axis.

For any $x_{2}^{0}$, the curve defined by equation (5) is symmetric with respect to $x_{1}$-axis; so we consider only the curves

$$
x_{2}=\sqrt{\left(x_{2}^{0}\right)^{2}-2\left(1-\cos x_{1}\right)} .
$$

If the following inequality

$$
\left(x_{2}^{0}\right)^{2}>2\left(1-\cos x_{1}\right)
$$

is satisfied, that is if the values of $x_{2}^{0}$ are such that $\left(x_{2}^{0}\right)^{2}>4$, then the range of $x_{1}$ is unrestricted and the curve defined by equation (6) is $2 \pi$-periodic graph over the $x_{1}$-axis. There are no equilibria on these curves and they correspond to the orbits of the motions of the pendulum with initial velocity so large that the pendulum revolves around and around without end.

As the behavior of this kind of orbits is well known for $\varepsilon=0$, here our purpose is to study the behavior of these orbits when the pendulum is forced, that is in the case $\varepsilon \neq 0$, by means of numerical methods.

\section{The rotation number for the forced pendulum}

For our purpose we resort to the rotation number $\rho$, which is well-known to have strong implications for dynamical systems (e.g. [5]). Its relevance for the study of the considered orbits of the forced pendulum can be summed up in the following way:

- If a unique $\rho$ exists and is rational, then the related orbit is periodic.

- If a unique $\rho$ exists and is irrational, then exists an invariant set of rotation number $\rho$.

- If a unique $\rho$ does not exist, then the forced pendulum exhibits topological chaos, i.e. positive topological entropy [1]; for more details see [6].

We remark that the last statement holds at least for circle maps [5]; however the considered orbit of the forced pendulum is topologically equivalent to a circle map, as it will be enlightened in the next Section.

Definition 1. (Poincaré-1885) For a monotone map $g: \mathbb{R} \rightarrow \mathbb{R}$ with $g(\theta+1)=$ $g(\theta)+1$

$$
\rho=\lim _{n \rightarrow \infty}\left(g^{n}(\theta)-\theta\right) / n
$$

exists for all $\theta \in \mathbb{R}$ and is independent of $\theta$ and is called rotation number of $g$. 
At first we consider $\varepsilon=0$ in (3),(4), that is we deal with a simple pendulum.

As pointed out in the previous Section, $x_{1}$ is the displacement angle of the pendulum which, in absence of friction, is a monotone (increasing) function of the time. When we numerically integrate (3), (4) with step size $h=1$, with any initial conditions, say $x_{1}(0), x_{2}(0)$, we obtain the solutions $x_{1}(n), x_{2}(n)$, $n=1,2,3,4, \ldots$ In the following we will assume $x_{1}(0)=0$ without loss of generality. For physical reasons, two solutions with the same $x_{2}$ that differ in $x_{1}$ by $2 \pi$, have to be considered the same (actually equations $(3),(4)$ remain the same under the change of variables $\left.\left(x_{1}, x_{2}\right) \rightarrow\left(x_{1}+2 \pi, x_{2}\right)\right)$; therefore if we normalize the period to 1 considering $f^{n}(0)=x_{1}(n) / 2 \pi, n=1,2,3 \ldots$, it can be viewed as the $n-t h$ iteration of a monotone map with $\theta=x_{1}(0)=0$ satisfying Definition 1 . Therefore when the pendulum is simple the rotation number always exists and is unique.

What happens when $\varepsilon \neq 0$ ? It is known that for the forced pendulum the limit providing the rotation number does not always exist; therefore two main questions arise:

1. when does the rotation number exist for the forced pendulum with a given parameter $\varepsilon$ ?

2. if the rotation number exists for the forced pendulum with given initial conditions and a given parameter $\varepsilon$, is it the same as for the simple pendulum with the same initial conditions? does it remains rational or irrational?

Here we answer at once the first question, whereas we will deal with the second question in Section 4, where numerical examples will provide numerical evidence about the answer.

If we choose as initial conditions $x_{1}(0)=0, x_{2}(0)=x_{2}^{0}>2$ and $0<\varepsilon<<1$, as already pointed out, the numerical integration of $(3),(4)$ allows to compute the rotation number $\rho$ if and only if the following finite limit exists

$$
\lim _{n \rightarrow \infty} \frac{x_{1}(n)}{2 \pi n}=\rho
$$

Obviously the used integration stepsize is much less than 1 .

We remark that we always have $\rho \neq 0$ and positive

Then we can derive a Numerical Convergence Condition.

Condition.If numerically integrating equations (3),(4) with given initial conditions and given parameter $\varepsilon$, we always obtain non-decreasing values for the displacement angle $x_{1}(n) / 2 \pi$ for increasing $n$ until $n$ is "large enough", then a unique rotation number $\rho$ does exist.

Proof. At first, we remark that the sequence $\frac{x_{1}(n)}{2 \pi} \frac{1}{n}$ has a finite limit $\rho$, when $n \rightarrow \infty$, if and only if the non-decreasing sequence $\frac{x_{1}(n)}{2 \pi}$ has a linear increasing asymptotic behavior, that is if $\frac{x_{1}(n)}{2 \pi} \rightarrow \rho n$ when $n \rightarrow \infty$. Actually, if the sequence $\frac{x_{1}(n)}{2 \pi}$ is bounded, then the sequence $\frac{x_{1}(n)}{2 \pi} \frac{1}{n}$ converges to 0 , and this 
means that the rotation number does not exist. Therefore, if $\frac{x_{1}(n+1)}{2 \pi}>\frac{x_{1}(n)}{2 \pi}$, for any considered $n$, the convergence of $\frac{x_{1}(n)}{2 \pi} \frac{1}{n}$ to a finite (nonnull) limit can be achieved.

Moreover, we remark that from the numerical point of view, we have to know the accuracy of the results obtained by means of the used numerical integration algorithm, say $\eta$; therefore if $\frac{x_{1}(n+1)}{2 \pi}-\frac{x_{1}(n)}{2 \pi}>\eta$ for any $n$, then the rotation number does exists.

In other words we can state that:

If numerically integrating equations (3),(4) with given initial conditions and given parameter $\varepsilon$, we always obtain non-decreasing values for the displacement angle $x_{1}(n) / 2 \pi$ for increasing $n$ until $n$ is "large enough", then topological chaos does not appear in the orbits of the considered forced pendulum.

Obviously we cannot carry out the numerical integration until $n$ is as large as we want, but only until $n$ is "large enough" and we remind that the determination of when $n$ is "large enough" is a questionable point (see Section 4).

\section{Numerical computation of the rotation number}

From a numerical point of view, it is clear that the main problems are:

- numerical integration of equations (3),(4) over a long time interval,

- numerical estimation of the limit (9), under the condition that it exists,

Let us treat the problems separately.

From the previous Section, it is clear that the numerical method used to integrate $(3),(4)$ is definitively relevant. As here we deal with numerical integration of a perturbed Hamiltonian system over a long time interval (i.e. $n>>1$ ), we choose a symplectic method such as the implicit midpoint method, which is second order, symmetric, and $A$-stable. This choice allows the numerical discretization to retain the property of symplectic map provided by the considered Hamiltonian system. Indeed the failure of well-known methods in mimicking Hamiltonian dynamics is due to the fact that they do not preserve symplecticity. In practice the midpoint solution is guaranteed to lie on the same orbit as the exact solution, whereas for example classical fourth order Runge-Kutta method does not; this fact is absolutely important for our kind of numerical problem, as we will see in the following. However here we will not give more details about symplectic methods (see e.g. [11], [4]).

In order to provide a numerical estimation of the limit (9), that is to numerically compute the rotation number, we resorted to some different methods, each of whom uses a different approach to the numerical problem.

\section{1 - Method Ml}

Under the condition that the limit (9) exists, the ratio $\frac{x_{1}(n)}{2 \pi n}$ tends to be a constant, when $n$ is large enough. Therefore Method $\mathbf{M l}$ computes the numerical sequence $\frac{x_{1}(n)}{2 \pi n}$, which provides a numerical approximation of the rotation number when $\frac{x_{1}(n)}{2 \pi n}$ tends to remain constant for increasing $n$. As already pointed out, 
we cannot know a priori when $n$ is large enough; in practice we make sure that, if for example $\eta=0.5 \cdot 10^{-3}$, for small values of $i$ we have $\left|\frac{x_{1}(n+i)}{2 \pi(n+i)}-\frac{x_{1}(n)}{2 \pi n}\right|<\eta$; this means that all the subsequent values coincide with $\frac{x_{1}(n)}{2 \pi n}$ at least in three decimal digits which are consequently considered three correct digits in the exact value of the limit $\rho$

\section{2 - Method Mg}

When $n$ is large enough (with the same caution as above), we have $\frac{x_{1}(n)}{2 \pi} \simeq \rho n$. Therefore for a fixed large $N$, Method $\mathbf{M g}$ computes the linear least square approximation of $x_{1}(n) / 2 \pi, 0 \leq n \leq N$; from the angular coefficient of the best fitting line we obtain an approximation of the rotation number.

3 - Method Mp ([7], [8])

It exploits the order by which the iterates of a diffeomorphism of the circle are generated and is based on two theorems which can be summed up in the following

Theorem 1. Let $f$ be a circle map with rotation number $\rho$; for each integer $n>0$, we call $X_{1}=\left\{f\left(X_{0}\right)\right\}, \ldots, X_{n}=\left\{f\left(X_{n-1}\right)\right\}=\left\{f^{n}\left(X_{0}\right)\right\}$ the fractional parts of the first $n$ iterates of $X_{0}$. Then for each $N>0$, the geometric order of the set of $X_{i}=\left\{f^{i}\left(X_{0}\right)\right\}, 1 \leq i \leq N$, allows to define four integers $A, a, B, b$ which are consecutive terms of the Farey sequence (therefore $|A b-a B|=1$ ) and provide the value of the rotation number $\rho$ by means of one of the following expressions

a) if $\rho$ is irrational then $A / a<\rho<B / b$;

b) if $\rho$ is rational (unreducible), say $\rho=p / q$ and $N<q$, then $A / a<\rho<B / b$;

c) if $\rho$ is rational (unreducible), say $\rho=p / q$, and $N \geq q$, then either $A / a=$ $\rho<B / b$ or $A / a<\rho=B / b$.

In all the cases $\Delta=|A / a-B / b|$ is such that $1 / N^{2}<\Delta<1 / N$.

In order to use this theorem, we need to clarify two points.

Remark 1. Equations (3) and (4) remain the same under the change of variable $x_{1}(t)=x_{1}(t+2 \pi)$; in such a way the orbit takes place on the circle $\mathbb{R} / 2 \pi \mathbb{Z}$, where the diffeomorphism of the circle $x_{1}(t)$ is defined.

Remark 2. The rotation number depends on the geometric order only; therefore it does not depend on the circle $\mathbb{R}, / k Z Z$, where $k$ is any real number. Actually the points of the orbits along circles with different $k$, can be obtained the ones from the others by means of a simple homotety.

Because of these two remarks, the previous Theorem which applies to maps of the circle of length 1, can be applied to maps of the circle of any length. For more details [8], [9], [10].

Actually the numerical approximation $\rho_{c}$ of an irrational rotation number $\rho$ is computed by the following expression:

$$
\rho_{c}=\frac{A+B}{a+b}
$$


Provided that methods $\mathbf{M l}$ and $\mathbf{M p}$ work only if the rotation number $\rho$ exists, it is easy to state the following Numerical Comparison Criterion.

Criterion. A unique rotation number exists if and only if all the three mentioned methods provide as a result the same numerical value within the used precision.

It is clear that all the presented methods are affected by the same integration errors due to (3), (4). Actually even though the used implicit midpoint method provides solutions lying on the exact orbit, it is not very accurate for $N$ large because of the accumulation of truncation errors. Therefore a major problem is the numerical error estimation $\Delta$ of the difference between the exact value of the rotation number $\rho$ and its numerical approximation $\rho_{c}$. Method $\mathbf{M g}$ can be considered the most reliable as it goes through a least square linear approximation of the behavior of $\frac{x_{1}(n)}{2 \pi}$ using linear polynomial $p(n)=s \cdot n+q$, where $q=0$ (as the polynomial goes through the origin); the computed value of parameter $s$ provides $\rho_{c}$. The advantage of this method is that it can be considered as a filter of the numerical integration errors. Moreover, as well-known, we can use the quantity $E=\sqrt{\frac{1}{n} \sum_{i=1}^{n}\left[\frac{x_{1}(n)}{2 \pi}-p(n)\right]^{2}}$ as an estimation of the accuracy of the method; indeed when $E=0$, it means that all th $\frac{x_{1}(n)}{2 \pi}$ lie on the line $p(n)$, for all the considered $n$.

Method Ml provides an error estimation only in the sense seen before. In practice if we assume for example $\eta=0.5 \cdot 10^{-3}$, and we have that there exists a $\bar{N}$ such that for all $N \geq \bar{N}$, we have $\left|\frac{x_{1}(N+1)}{2 \pi(N+1)}-\frac{1(N)}{2 \pi N}\right|<0.5 \cdot 10^{-3}$, then all the subsequent values coincide with $\frac{x_{1}(\bar{N})}{2 \pi \bar{N}}$ at least in the first three decimal digits. This allows us to state that these digits are even the first three correct digits in $\rho$.

A major drawback of methods $\mathbf{M g}$ and $\mathbf{M l}$ is that they do not allow to detect whether the rotation number is rational or irrational, whereas another drawback is that their rate of convergence cannot be known a priori, even though from a theoretical point of view, it is known to be less than linear.

On the contrary method $\mathbf{M p}$ can distinguish between rational and irrational rotation number and in the case of rational rotation number its error is equal to 0 .

Method Mp provides an accurate error estimation. The method is based on a normalized continued fraction expansion of the rotation number. In literature other methods based on continued fraction expansions were presented (see [2] and [5]); however the rate of convergence can vary significantly when different continued fraction approximations are used. For example when the rotation number is a Liouville number, the convergence of method in [5] is so slow that it becomes questionable from a numerical point of view to achieve 7 significant digits, whereas method $\mathbf{M p}$ converges very fast. From a theoretical point of view we can guarantee that method $\mathbf{M p}$ approximates any irrational rotation number 
with an accuracy between $1 / N$ and $1 / N^{2}$, but experimentally we found that the rate of convergence is usually very close to be quadratic.

We remark that the only drawback of method $\mathbf{M p}$ is that when the four integers $A, a, B, b$ fail to be consecutive terms of the Farey sequence, due to machine accuracy, then the method is not reliable any longer; however as this event can be detected very easily (it happens when $A b-a B \neq \pm 1$ ), the method can be controlled, improving for example the numerical integration accuracy.

\section{Numerical examples}

Here we present some numerical examples, which are significant but far to be exhaustive.

In the following Tables we report the values of $\rho_{c}$ (rounded to 3 digits) for $x_{1}(0)=0, N=200$; the used integration step for the implicit midpoint rule was $h=0.005 ; \Delta_{M l}$ indicates the value of $\left|\frac{x_{1}(k+1)}{2 \pi k}-\frac{x_{1}(k)}{2 \pi k}\right|$ when $k=195$ and we experimentally checked that for all $k, 196 \leq k \leq 199$, such differences were $\leq \Delta_{M l}$; therefore $\Delta_{M l}$ can be considered an error estimation for method $\mathrm{Ml}$; $\Delta_{M p}$ refers to the actual error estimation by method $\mathbf{M p}$, therefore $\frac{1}{2}\left(\frac{B}{b}-\frac{A}{a}\right)$, $\Delta_{M g}$ reports the computed value of $E$, given above.

We emphasize that when the rotation number exists and is irrational, all the methods provide the same rounded value, as it is expected from Numerical Comparison Criterion. However only method $\mathbf{M p}$ provides the rational rotation number (see Table 1), even though in this case we need to use $N>209$.

Crosses indicate when the rotation number does not exist.

Now we can empirically answer the question 2 in Section 2: if the rotation number exists for the forced pendulum with given initial conditions and given $\varepsilon$, is it the same as for the simple pendulum with the same initial conditions?

From Table 3, we see that for given initial conditions, when $\varepsilon$ increases significantly, the rotation number changes slightly, but clearly, therefore for $\varepsilon>10^{-3}$ the numerical evidence provides a negative answer to the above question.

Table 1. $\varepsilon=0$

\begin{tabular}{lllllll}
\hline$x_{2}(0)$ & $\mathrm{Ml}$ & $\Delta_{M l}$ & $\mathrm{Mg}$ & $\Delta_{M g}$ & $\mathrm{Mp}$ & $\Delta_{M p}$ \\
2.005 & .124 & $4 \mathrm{E}-4$ & .124 & $1 \mathrm{E}-1$ & .124 & $3 \mathrm{E}-5$ \\
2.2 & .237 & $2 \mathrm{E}-4$ & .237 & $5 \mathrm{E}-2$ & .237 & $8 \mathrm{E}-5$ \\
2.4 & .290 & $4 \mathrm{E}-5$ & .290 & $3 \mathrm{E}-2$ & .290 & $8 \mathrm{E}-5$ \\
2.6 & .335 & $3 \mathrm{E}-4$ & .335 & $2 \mathrm{E}-2$ & $70 / 209$ & 0 \\
2.8 & .376 & $2 \mathrm{E}-4$ & .376 & $2 \mathrm{E}-2$ & .376 & $2 \mathrm{E}-5$ \\
3.0 & .414 & $2 \mathrm{E}-4$ & .414 & $2 \mathrm{E}-2$ & .414 & $3 \mathrm{E}-5$ \\
3.2 & .452 & $7 \mathrm{E}-5$ & .452 & $1 \mathrm{E}-2$ & .452 & $8 \mathrm{E}-5$ \\
\hline
\end{tabular}


Table 2. $\varepsilon=0.1$

\begin{tabular}{lllllll}
\hline$x_{2}(0)$ & $\mathrm{Ml}$ & $\Delta_{M l}$ & $\mathrm{Mg}$ & $\Delta_{M g}$ & $\mathrm{Mp}$ & $\Delta_{M p}$ \\
2.005 & - & - & $-2 \mathrm{E}-4$ & $3 \mathrm{E}-1$ & - & - \\
2.2 & - & - & $-1 \mathrm{E}-2$ & 3.1 & - & - \\
2.4 & .272 & $5 \mathrm{E}-4$ & .272 & $4 \mathrm{E}-2$ & .272 & $3 \mathrm{E}-4$ \\
2.6 & .320 & $4 \mathrm{E}-4$ & .320 & $1 \mathrm{E}-1$ & .320 & $7 \mathrm{E}-4$ \\
2.8 & .366 & $3 \mathrm{E}-4$ & .366 & $2 \mathrm{E}-2$ & .366 & $3 \mathrm{E}-4$ \\
3.0 & .406 & $3 \mathrm{E}-4$ & .406 & $2 \mathrm{E}-2$ & .406 & n.c. \\
3.2 & .445 & $2 \mathrm{E}-4$ & .445 & $1 \mathrm{E}-4$ & .445 & $3 \mathrm{E}-4$ \\
\hline
\end{tabular}

Table 3. $\varepsilon=0.1$

\begin{tabular}{llllllll}
\hline$x_{2}(0) \backslash \varepsilon$ & 0 & 0.001 & 0.005 & 0.01 & 0.05 & 0.1 & 0.5 \\
2.005 & .124 & .124 & $\times$ & $\times$ & $\times$ & $\times$ & $\times$ \\
2.2 & .237 & .237 & .235 & .234 & .217 & $\times$ & $\times$ \\
2.4 & .290 & .290 & .289 & .289 & .282 & .272 & $\times$ \\
2.6 & $70 / 209$ & .335 & .334 & .334 & .329 & .320 & $\times$ \\
2.8 & .376 & .376 & .375 & .375 & .371 & .366 & .318 \\
3.0 & .414 & .414 & .414 & .414 & .410 & .406 & .365 \\
3.2 & .452 & .452 & .452 & .451 & .448 & .445 & .412 \\
\hline
\end{tabular}

From Table 2 it is clear when we say that the rotation number does not exist; here "n.c." means that the error is not computable because method Mp fails to find four integers belonging to Farey sequence.

There is a numerical evidence that for each value of $x_{2}(0)$ there exists a value of $\varepsilon^{*}$ such that for $\varepsilon>\varepsilon^{*}$ the rotation number does not exist. The value of $\varepsilon^{*}$ does not increase linearly with $x_{2}(0)$. Moreover we can see that for any fixed value of $x_{2}(0)$ and for increasing values of $\varepsilon$ from 0 , the rotation number is always not increasing.

We emphasize that the existence of the rotation number is not affected by the "graphical" behavior of the orbits, in the sense that the rotation number exists even when orbits clearly split and do not overlap any longer. For example Fig. 1 and 2 show the orbits in the phase space for $x_{2}(0)=2.4$ and $\varepsilon=0.005$, 0.1 , respectively (see Table 3 ); in spite of what appears in Fig. 2, in both cases the rotation number exists and chaos does not happen; indeed as the rotation number depends on the geometric order of the iterates of the map, it means that a kind of geometric order is preserved, even though it is not immediately detectable from the graphical representation, as in Fig.2. On the other hand, Fig. 3 shows the orbits for $x_{2}(0)=2.4$ and $\varepsilon=0.5$, that is the case when the rotation number does not exist at all and chaos happens, as no geometric order is preserved any longer. In Table 3 bold characters indicate rotation numbers referring to these cases of splitted orbits, whose rotation number exists anyway. 
We point out that this new fact can be interesting to KAM theory and can be detected here just because we have approximated numerically the rotation numbers.

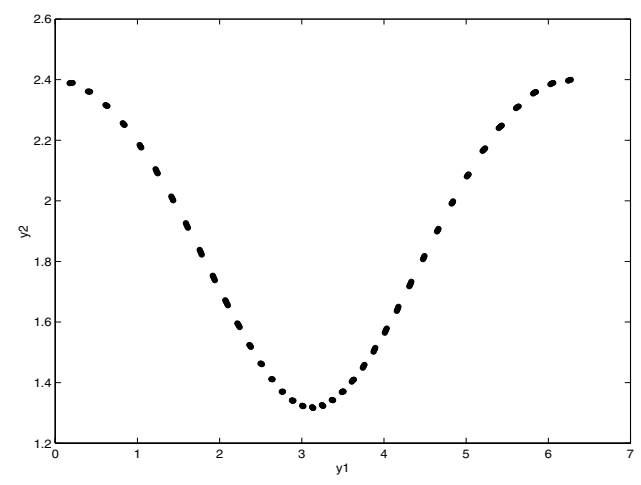

Fig. 1.

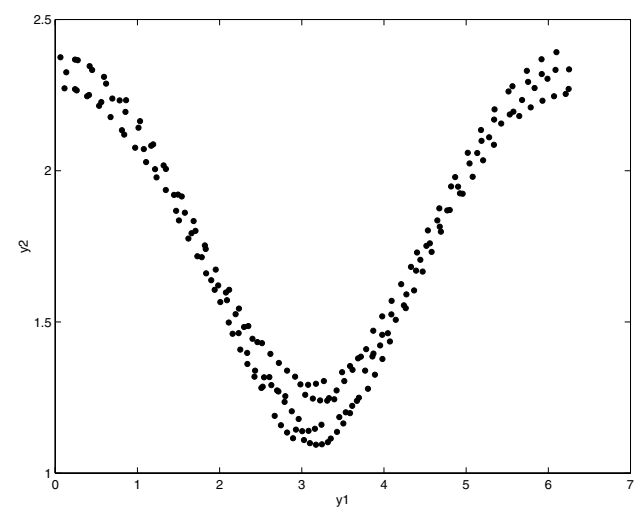

Fig. 2.

\section{Conclusion}

In [3] the rotation number was already presented as a quantitative measure of chaos, however the method given there to compute the rotation number did not provide any numerical result. Here referring to the forced pendulum, we numerically support their statement. Indeed we present a comparison among 


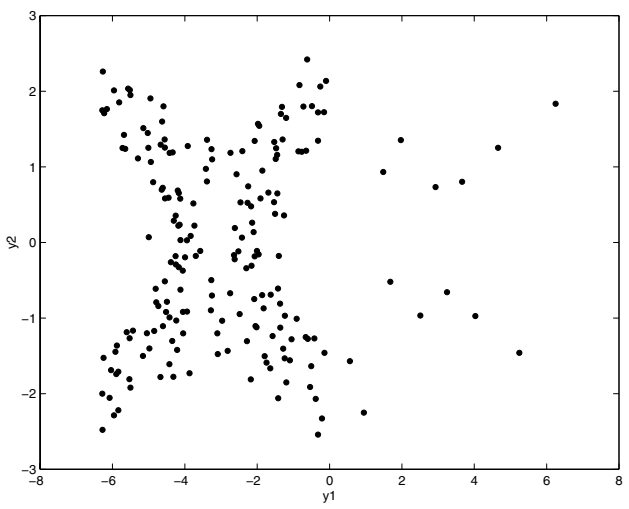

Fig. 3.

some different numerical methods and show how they can provide a reliable estimation of the rotation number, when it exists. Moreover we emphasize that the existence of the rotation number does not depend on the "graphical" behavior of the orbits, but on the geometric order of the iterates only. When the rotation number does not exist, topological chaos appears; therefore the rotation number can be used as a reliable quantitative index of chaos itself.

\section{References}

1. Block, L., Guckenheimer, J., Misiurewicz, M., Young, L. S.: Periodic points and topological entropy of one-dimensional maps. Springer Lecture Notes in Math. 819 (1980).

2. Bruin, H.: Numerical determination of the continued fraction expansion of the rotation number. Physica D 59 (1992) 158-168.

3. Gambaudo, J.M., Glendinning, P., Tresser, C.: The rotation interval as a computable measure of chaos.Physics Letters 105A 3, (1984) 97-100.

4. Hairer, E., Lubich, C., Wanner, G.: Geometric Numerical Integration. SpringerVerlag (to appear).

5. MacKay, R.S.: Rotation interval from a time series. J. Phys. A, 20 (1987), 587-592.

6. MacKay, R.S., Tresser, C.: Transition to topological chaos for circle maps. Physica D 19, (1986), 206-237.

7. Pavani, R., Talamo, R.: Conjugating the Poincaré-map to a rotation. Ann. Mat. Pura Appl. IV 166, (1994), 381-394.

8. Pavani, R.: A numerical approximation of the rotation number. Appl. Math. Comp. 73, (1995), 191-201.

9. Pavani, R.: The numerical approximation of the rotation number of planar maps. Computers Math. Appl. 33, (1997), 103-110.

10. Pavani, R.: Numerical study of the rotation number for the forced pendulum. Quad. Dip. Mat. Politecnico di Milano n.449/P (2001).

11. Sanz-Serna, J.M., Calvo, M.P.: Numerical Hamiltonian Problems. Chapman \& Hall, London New York (1994). 